\title{
Phytotoxic and Nematicidal Components of Lavandula luisieri
}

Luis F. Julio, ${ }^{\dagger}$ Alejandro F. Barrero** M. Mar Herrador del Pino,${ }^{\ddagger}$ Jesús F. Arteaga, ${ }^{\S}$

Jesús Burillo, ${ }^{\perp}$ Maria Fe Andres, ${ }^{\dagger}$ Carmen E. Díaz ${ }^{\perp}$ and Azucena González-Coloma*,†

${ }^{\dagger}$ Instituto de Ciencias Agrarias. Consejo Superior de Investigaciones Científicas.

Serrano 115-bis, 28006 Madrid, Spain

Departamento de Química Orgánica, Instituto de Biotecnología, Universidad de Granada, Campus de Fuente Nueva, s/n, 18071 Granada, Spain

${ }^{\S}$ CIQSO - Center for Research in Sustainable Chemistry and Department of Chemical

Engineering, Physical Chemistry, and Organic Chemistry, Facultad de Ciencias

Experimentales, Universidad de Huelva, Campus el Carmen, 21071 Huelva, Spain

${ }^{\perp}$ Departamento de Ciencia, Tecnología y Universidad, Centro de Investigación y

Tecnología Agroalimentaria de Aragón, Gobierno de Aragón, Avda Montañana, 930, Zaragoza, Spain

"Instituto de Productos Naturales y Agrobiología, Consejo Superior de Investigaciones Científicas. Avda. Astrofísico F. Sánchez, 3, 38206 La Laguna, Tenerife, Spain 


\section{Supporting information}

\section{List of contents}

Figure S1. ${ }^{1} \mathrm{H}-\mathrm{NMR}$ spectrum of compound $1\left(\mathrm{CDCl}_{3}, 500 \mathrm{MHz}\right)$

Figure S2. ${ }^{13} \mathrm{C}-\mathrm{NMR}$ spectrum of compound $\mathbf{1}\left(\mathrm{CDCl}_{3}, 125 \mathrm{MHz}\right)$

Figure S3. HREIMS of compound 1

Figure S4. ${ }^{1} \mathrm{H}-\mathrm{NMR}$ spectrum of compound $2\left(\mathrm{CDCl}_{3}, 500 \mathrm{MHz}\right)$

Figure S5. ${ }^{13} \mathrm{C}$-NMR spectrum of compound $2\left(\mathrm{CDCl}_{3}, 125 \mathrm{MHz}\right)$

Figure S6. HREIMS spectrum compound 2

Figure S7. ${ }^{1} \mathrm{H}-\mathrm{NMR}$ spectrum of compound $3\left(\mathrm{CDCl}_{3}, 500 \mathrm{MHz}\right)$

Figure S8. ${ }^{13} \mathrm{C}-\mathrm{NMR}$ spectrum of compound $3\left(\mathrm{CDCl}_{3}, 125 \mathrm{MHz}\right)$

Figure S9. HRESI-TOFMS of compound 3

Figure S10. ${ }^{1} \mathrm{H}-\mathrm{NMR}$ spectrum of compound $5\left(\mathrm{CDCl}_{3}, 500 \mathrm{MHz}\right)$

Figure S11. ${ }^{13} \mathrm{C}$-NMR spectrum of compound $5\left(\mathrm{CDCl}_{3}, 125 \mathrm{MHz}\right)$

Figure S12. HREIMS of compound 5

Figure S13. ${ }^{1} \mathrm{H}-\mathrm{NMR}$ spectrum of compound $\mathbf{6 a}\left(\mathrm{CDCl}_{3}, 500 \mathrm{MHz}\right)$

Figure S14. ${ }^{13} \mathrm{C}-\mathrm{NMR}$ spectrum of compound $\mathbf{6 a}\left(\mathrm{CDCl}_{3}, 125 \mathrm{MHz}\right)$

Figure S15. HREIMS of compound $\mathbf{6 a}$

Figure S16. ${ }^{1} \mathrm{H}-\mathrm{NMR}$ spectrum of compound $7\left(\mathrm{CDCl}_{3}, 500 \mathrm{MHz}\right)$

Figure S17. ${ }^{13} \mathrm{C}$-NMR spectrum of compound $7\left(\mathrm{CDCl}_{3}, 125 \mathrm{MHz}\right)$

Figure S18. HREIMS of compound 7

Figure S19. ${ }^{1} \mathrm{H}-\mathrm{NMR}$ spectrum of compound $\mathbf{8}\left(\mathrm{CDCl}_{3}, 500 \mathrm{MHz}\right)$

Figure S20. ${ }^{13} \mathrm{C}$-NMR spectrum of compound $\mathbf{8}\left(\mathrm{CDCl}_{3}, 125 \mathrm{MHz}\right)$

Figure S21. HREIMS of compound 8

Table S1. Phytotoxic Effects of L. luisieri extracts

Table S2. Antifeedant and Nematicidal Effects of $L$. luisieri extracts 


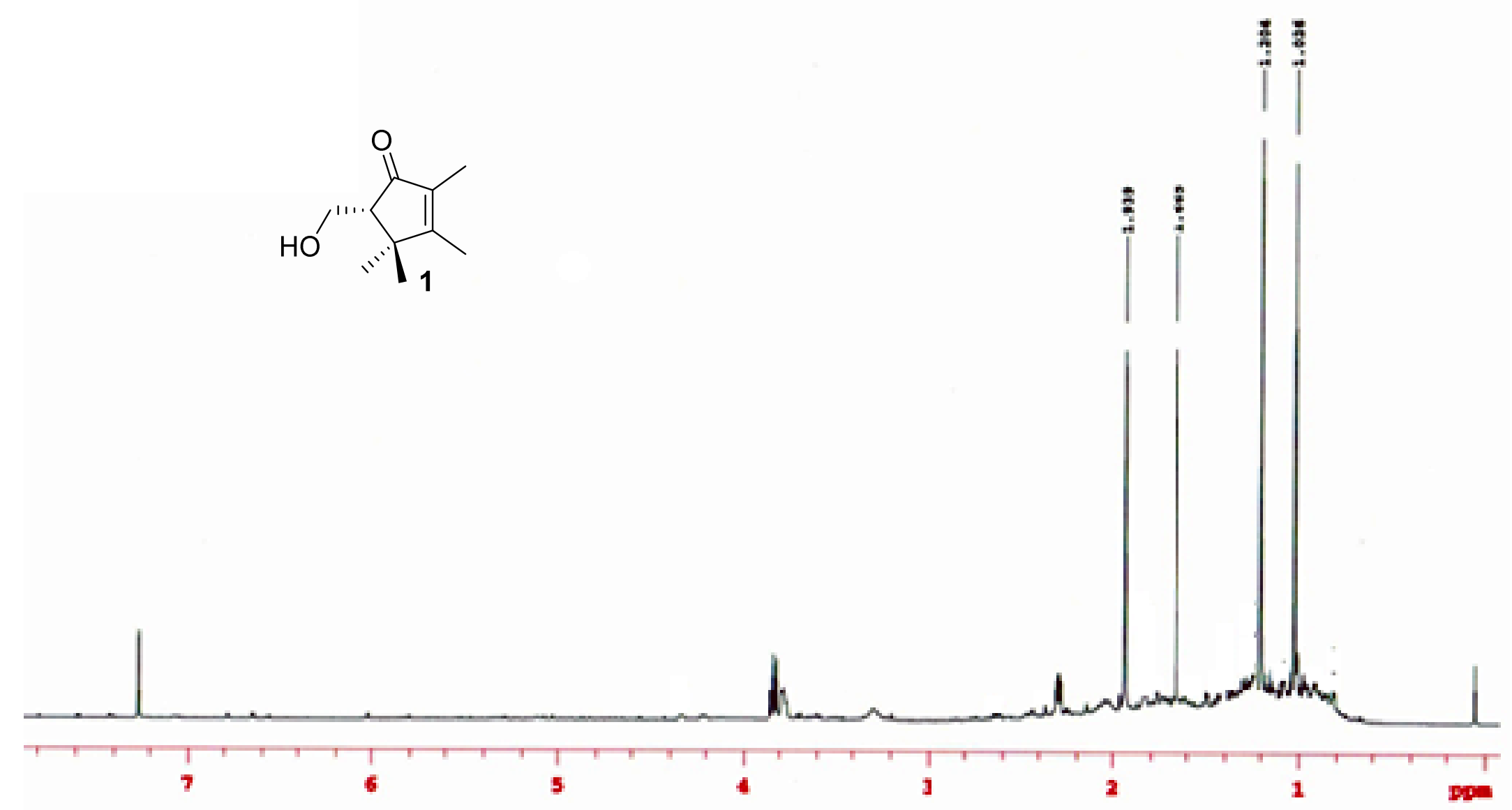

Figure S1. ${ }^{1} \mathrm{H}-\mathrm{NMR}$ spectrum of compound $\mathbf{1}\left(\mathrm{CDCl}_{3}, 500 \mathrm{MHz}\right)$. 


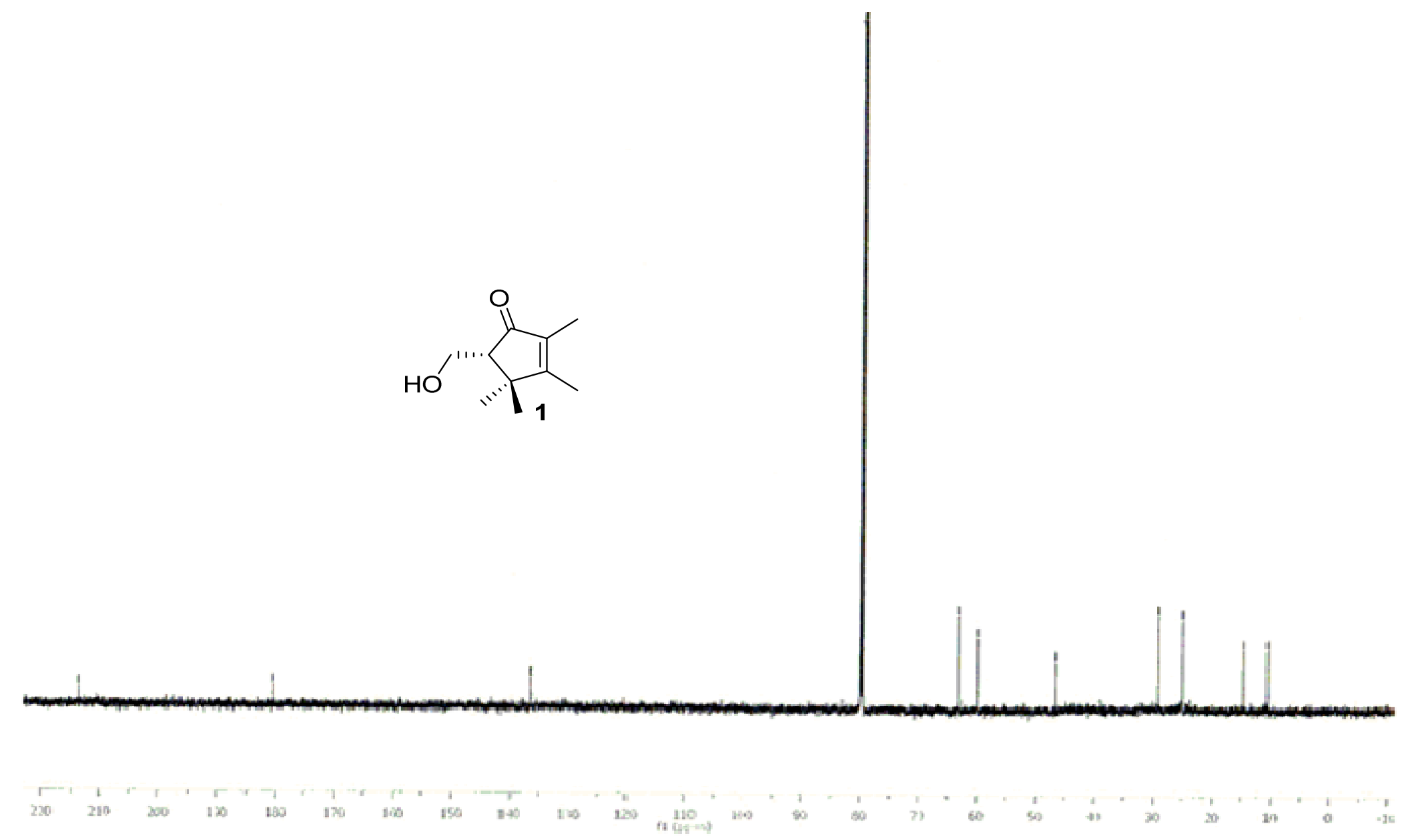

Figure S2. ${ }^{13} \mathrm{C}-\mathrm{NMR}$ spectrum of compound $\mathbf{1}\left(\mathrm{CDCl}_{3}, 125 \mathrm{MHz}\right)$. 


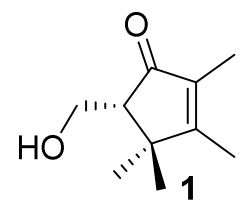

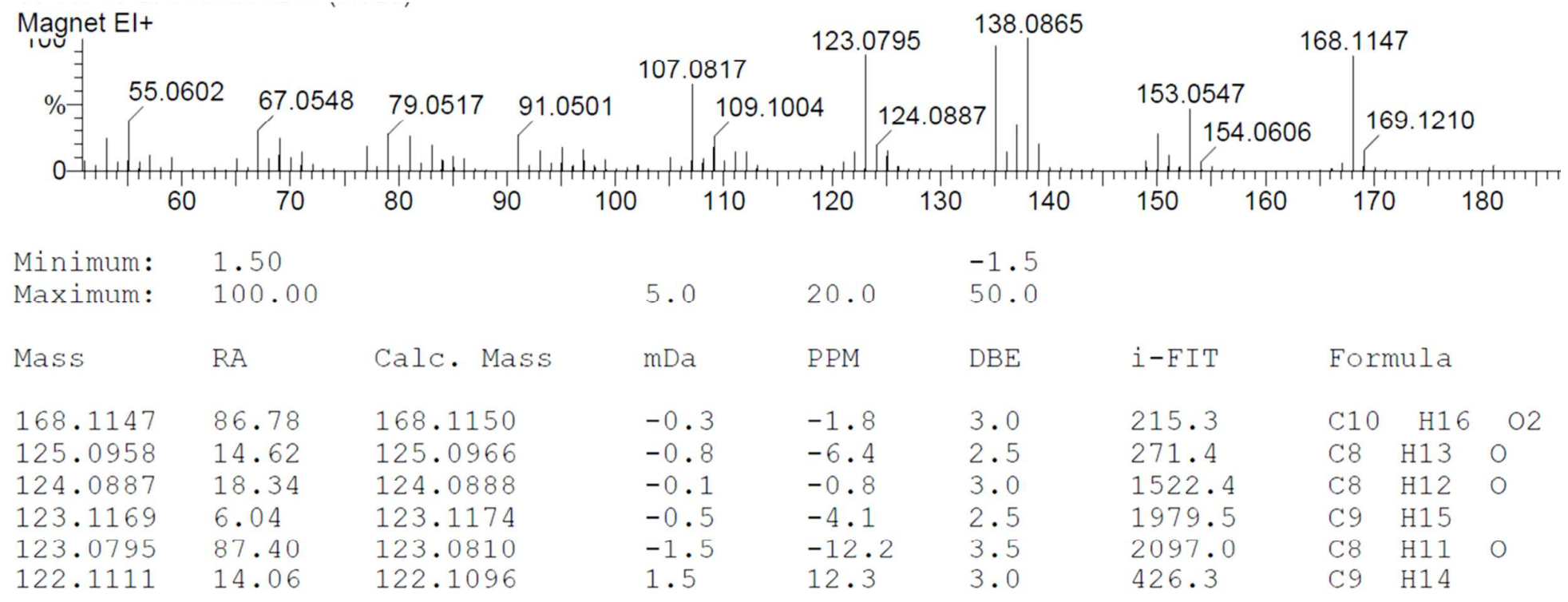

Figure S3. HREIMS of compound 1. 


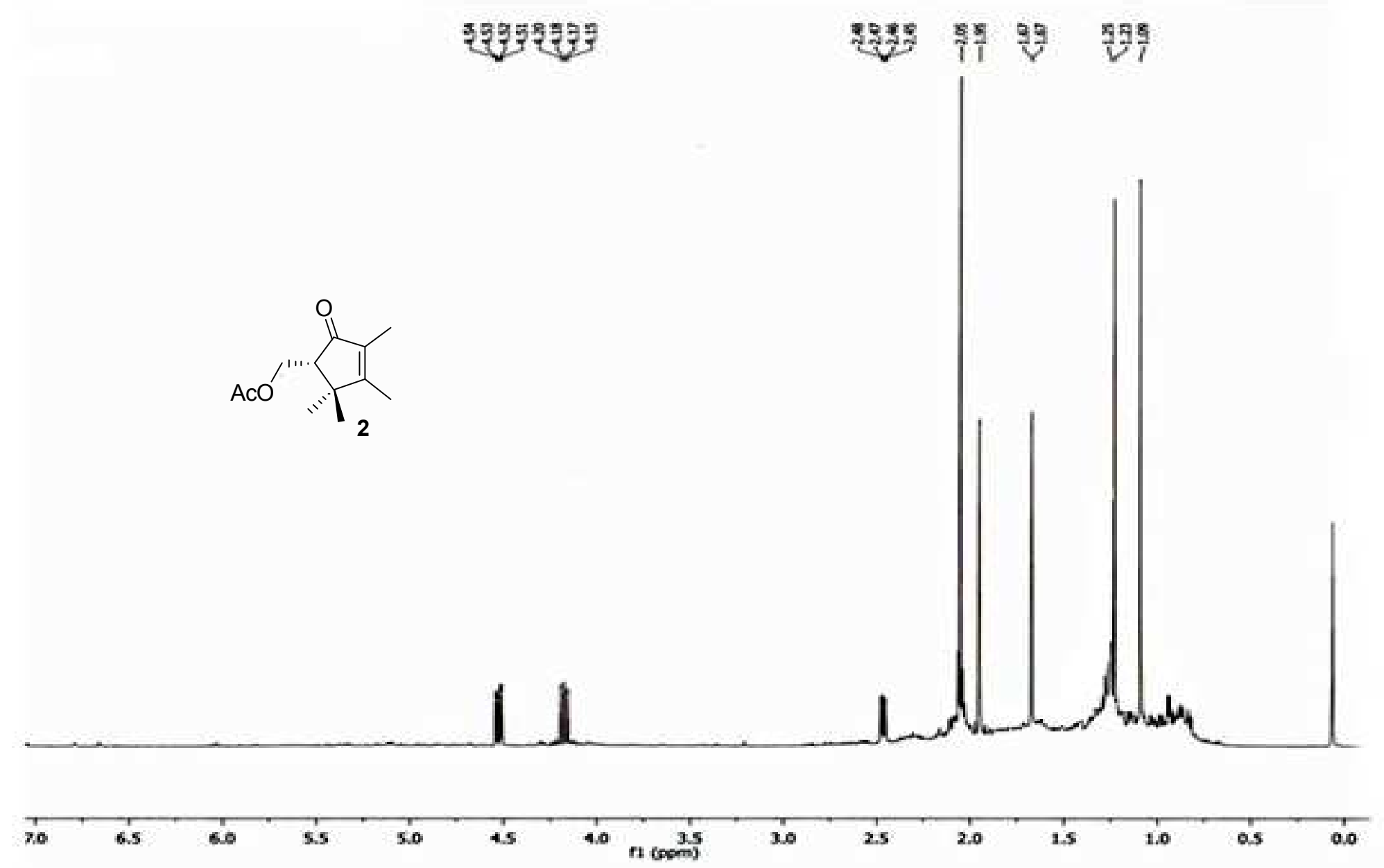

Figure S4. ${ }^{1} \mathrm{H}-\mathrm{NMR}$ spectrum of compound $2\left(\mathrm{CDCl}_{3}, 500 \mathrm{MHz}\right)$. 

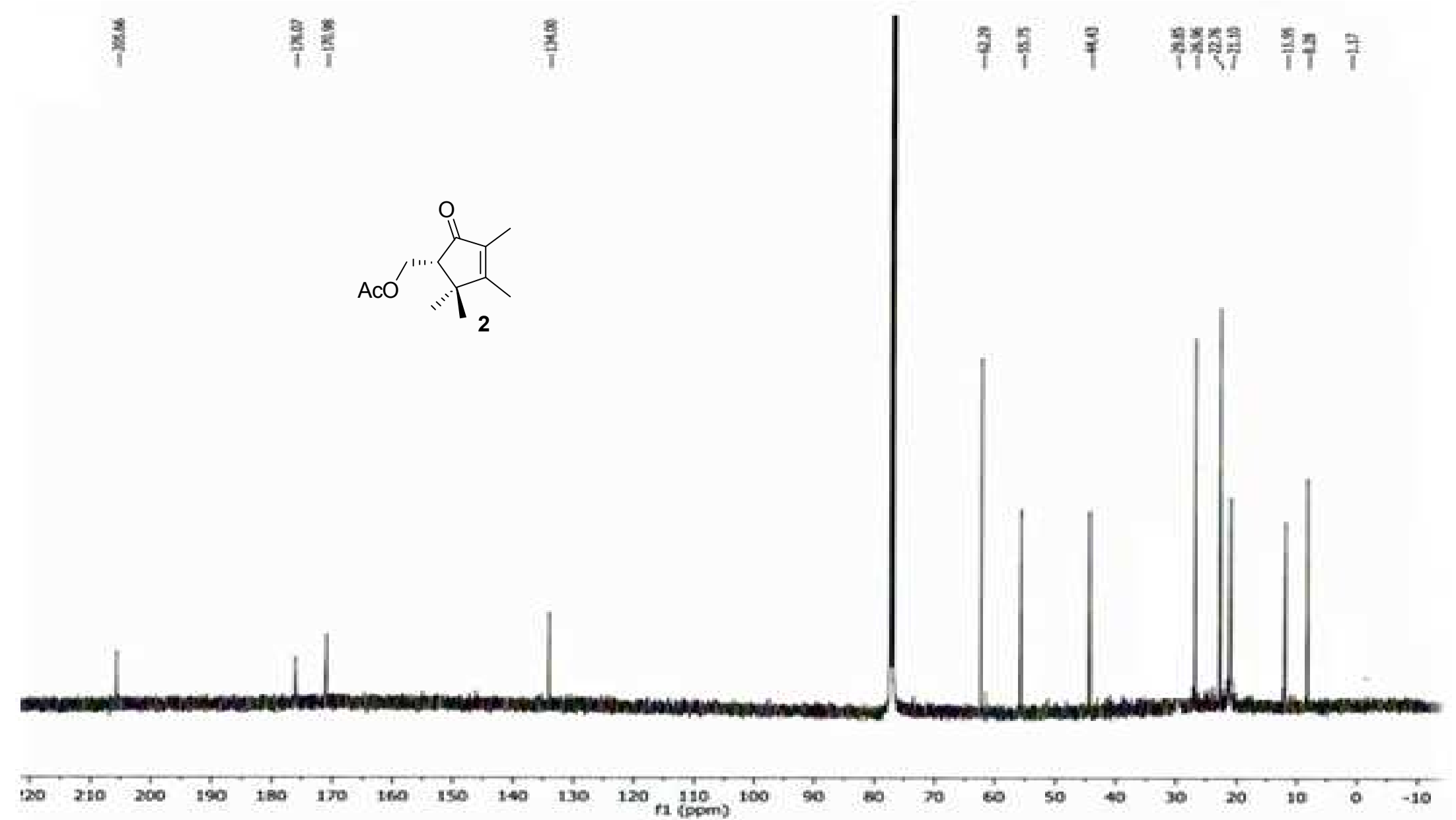

Figure S5. ${ }^{13} \mathrm{C}-\mathrm{NMR}$ spectrum of compound $2\left(\mathrm{CDCl}_{3}, 125 \mathrm{MHz}\right)$. 


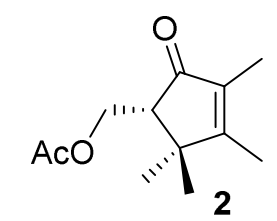

Magnet El+
Minimum:
10.00

Figure S6. HREIMS of compound 2. 


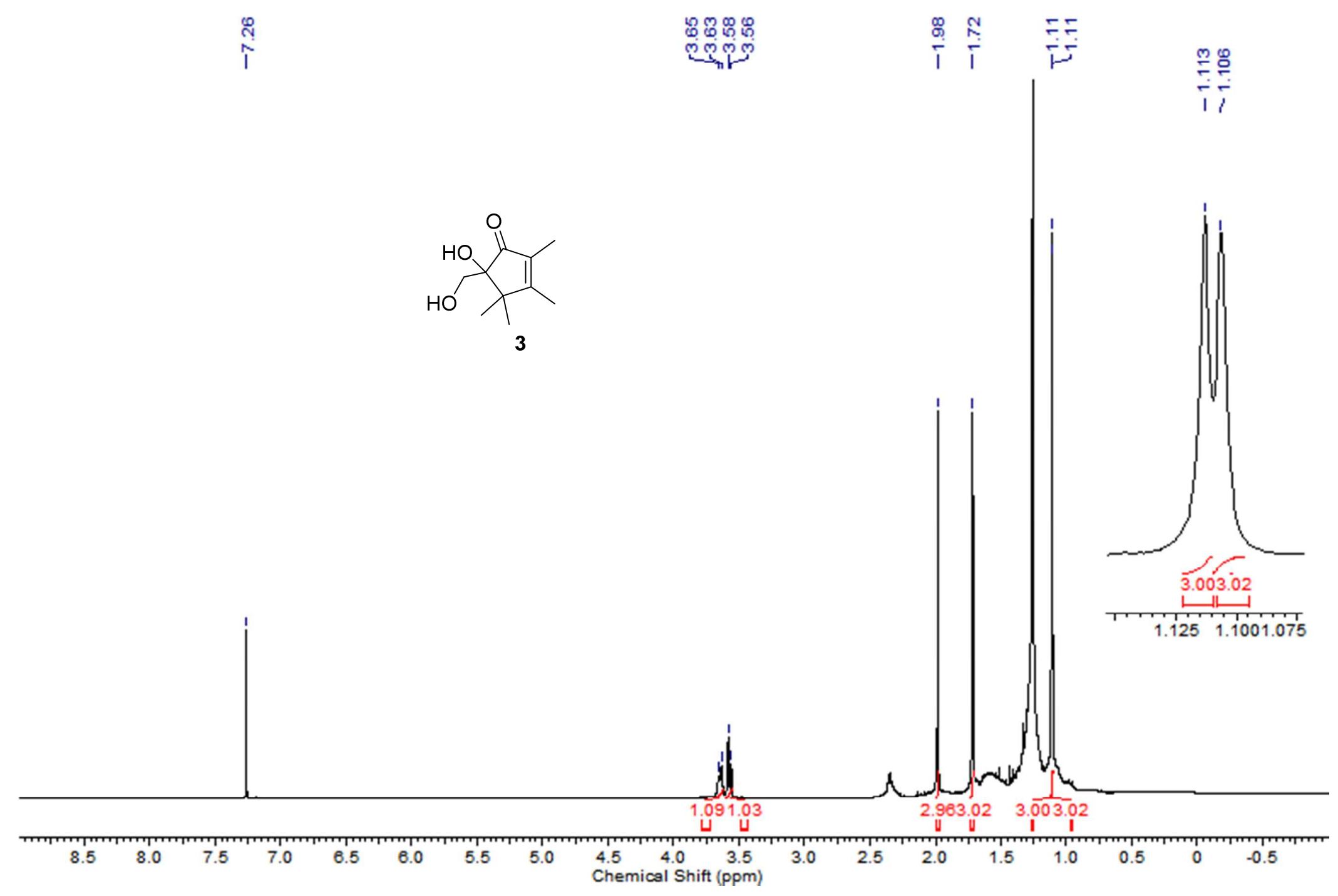

Figure S7. ${ }^{1} \mathrm{H}-\mathrm{NMR}$ spectrum of compound $3\left(\mathrm{CDCl}_{3}, 500 \mathrm{MHz}\right)$. 

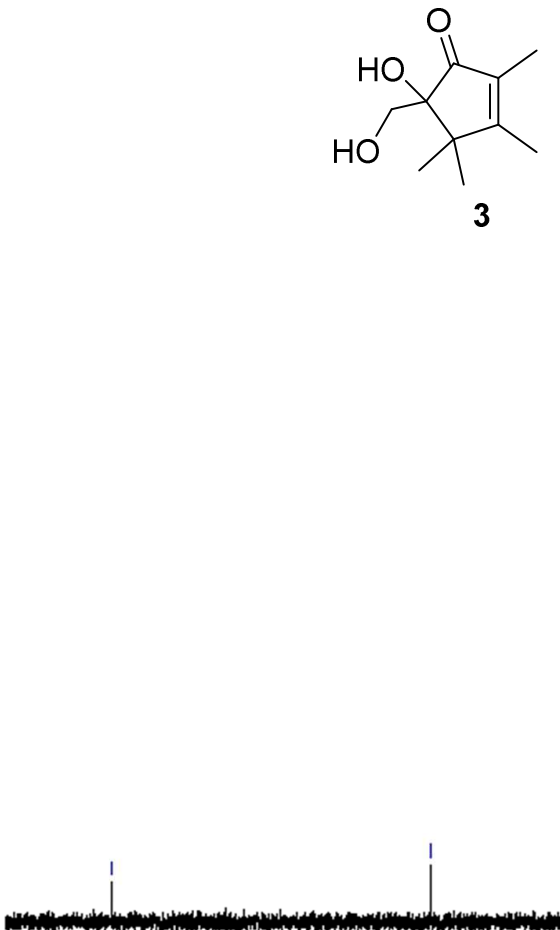

Figure S8. ${ }^{13} \mathrm{C}-\mathrm{NMR}$ spectrum of compound $3\left(\mathrm{CDCl}_{3}, 125 \mathrm{MHz}\right)$. 


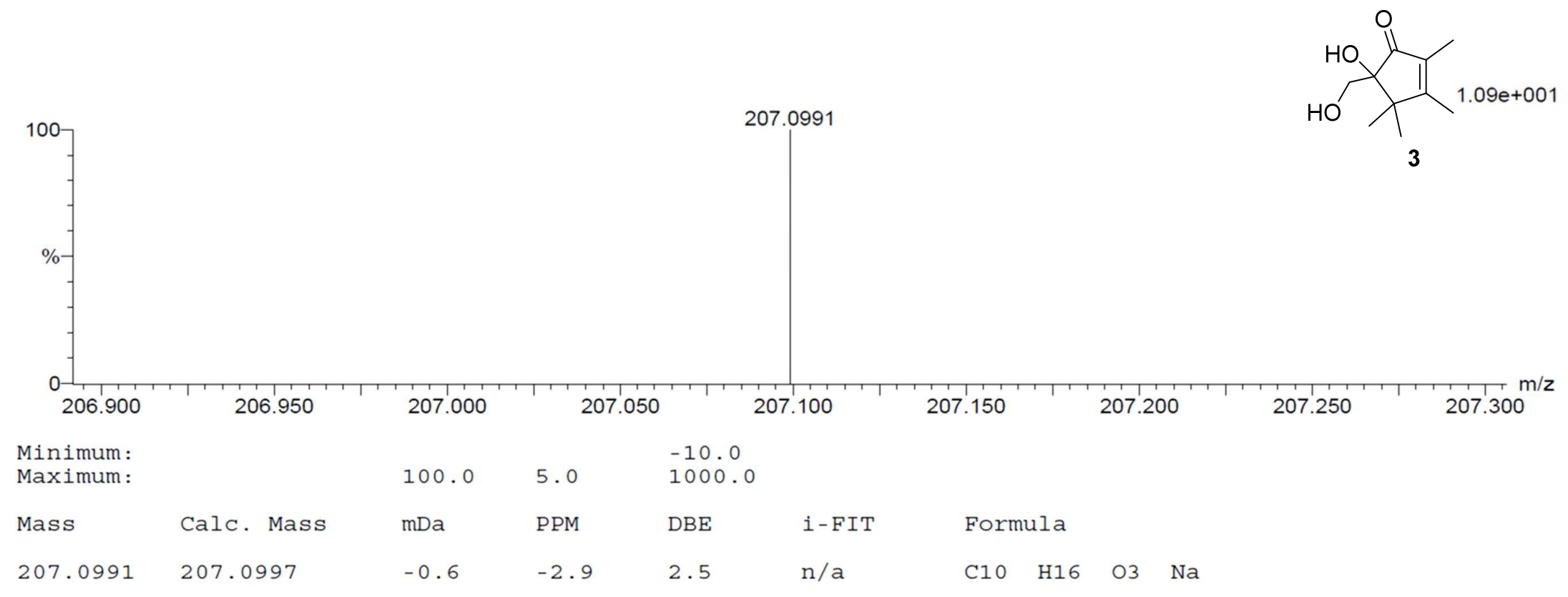

Figure S9. HRESI-TOFMS of compound 3. 


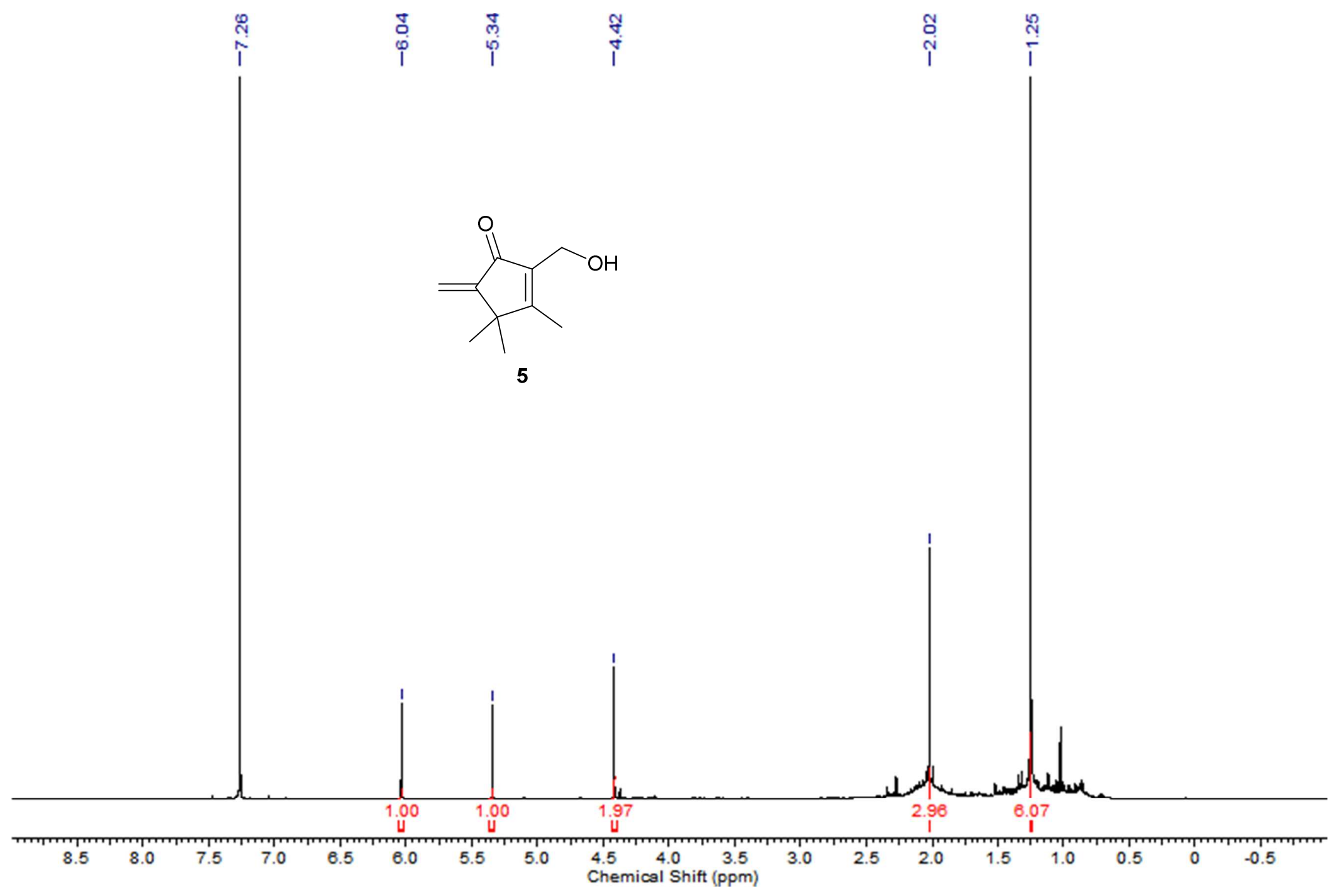

Figure S10. ${ }^{1} \mathrm{H}-\mathrm{NMR}$ spectrum of compound $5\left(\mathrm{CDCl}_{3}, 500 \mathrm{MHz}\right)$. 


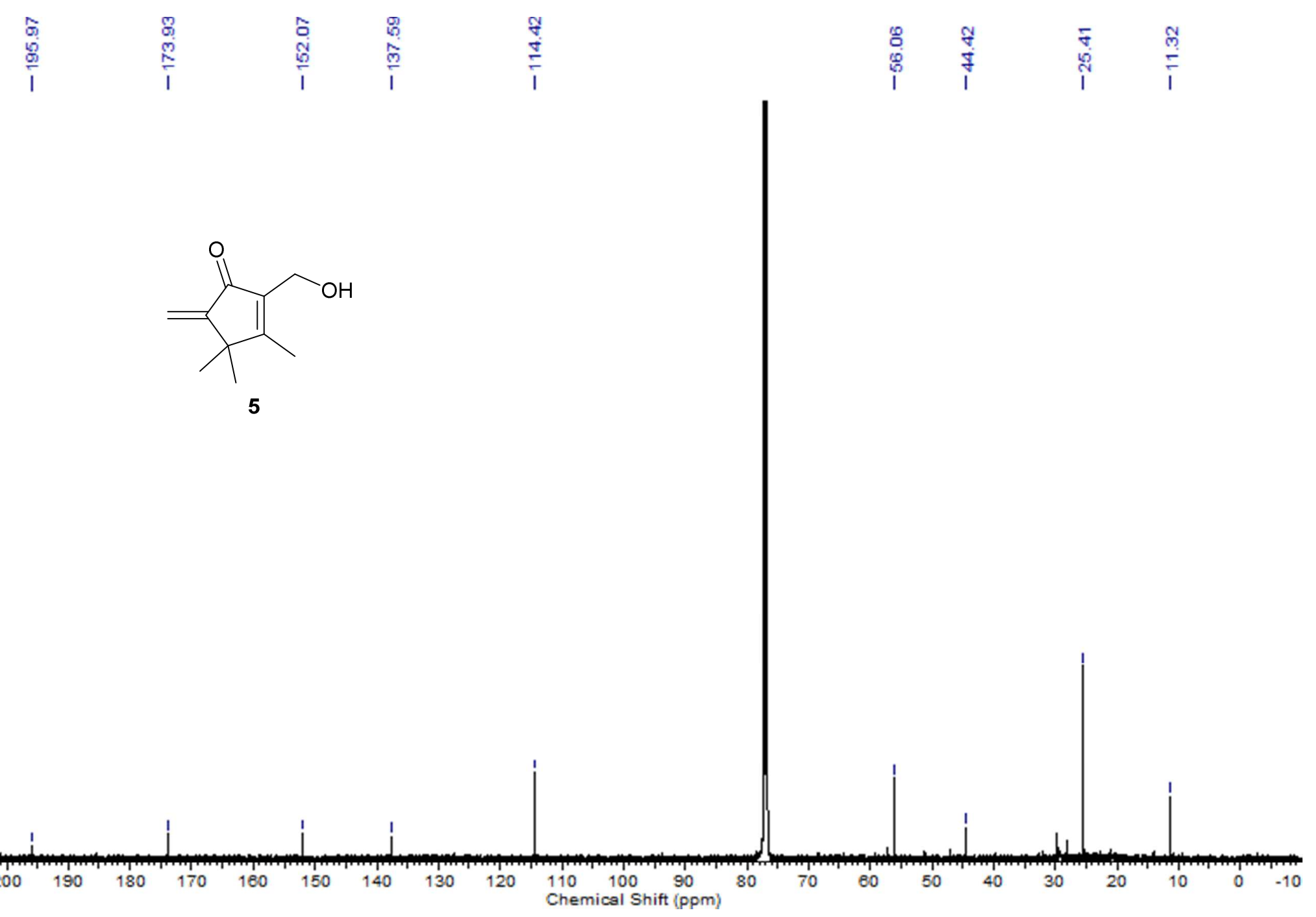

Figure S11. ${ }^{13} \mathrm{C}-\mathrm{NMR}$ spectrum of compound $\mathbf{5}(\mathrm{CDCl} 3,125 \mathrm{MHz})$. 


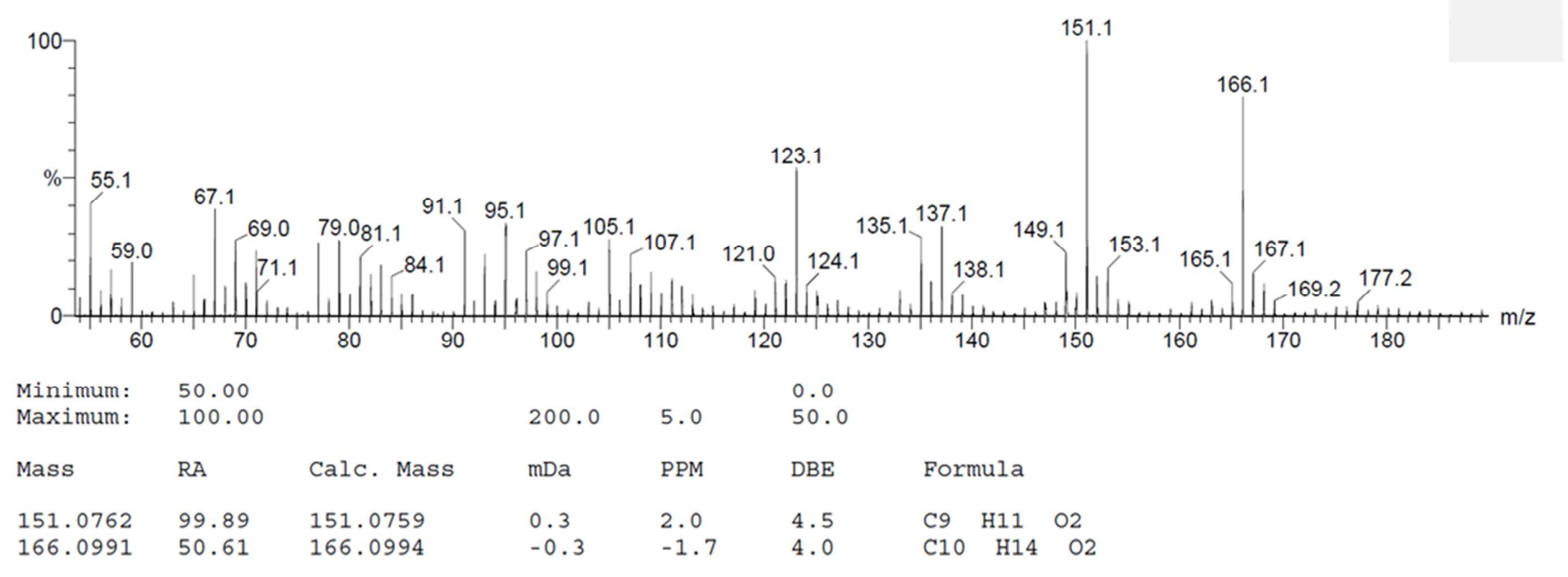

Figure S12. HREIMS of compound 5. 


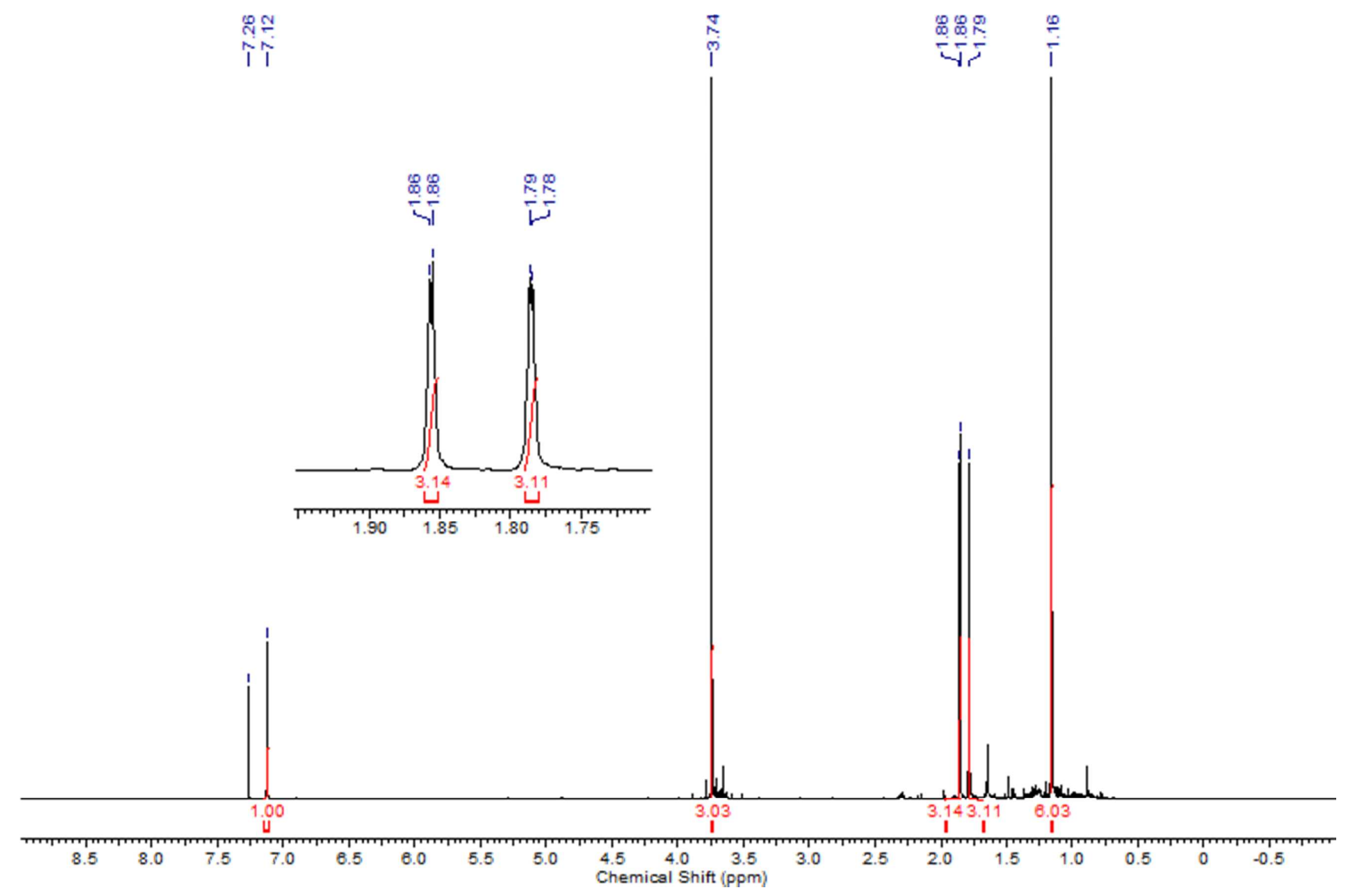

Figure S13. ${ }^{1} \mathrm{H}-\mathrm{NMR}$ spectrum of compound $\mathbf{6 a}\left(\mathrm{CDCl}_{3}, 500 \mathrm{MHz}\right)$. 


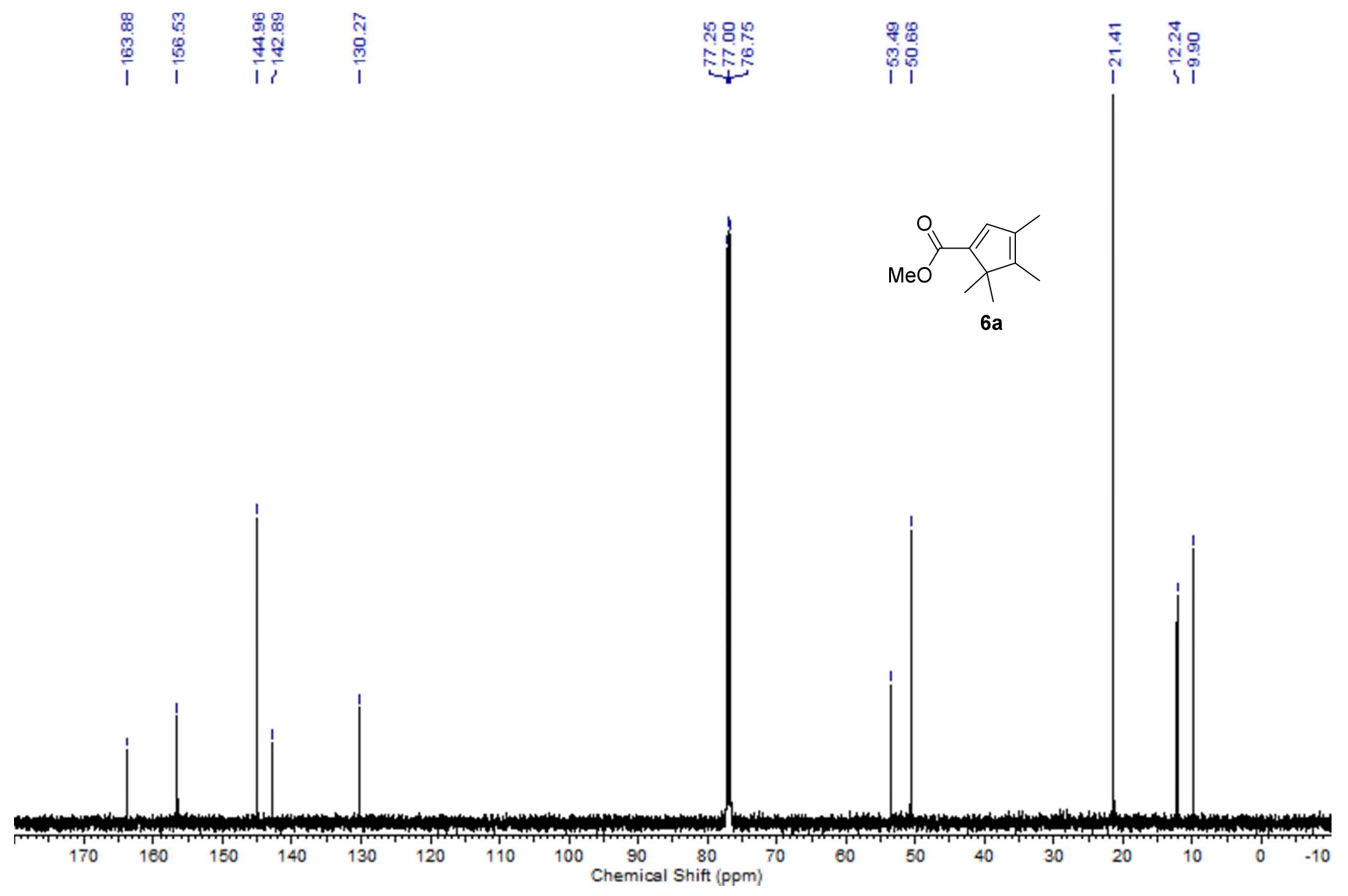

Figure S14. ${ }^{13} \mathrm{C}-\mathrm{NMR}$ spectrum of compound $\mathbf{6 a}\left(\mathrm{CDCl}_{3}, 125 \mathrm{MHz}\right)$. 

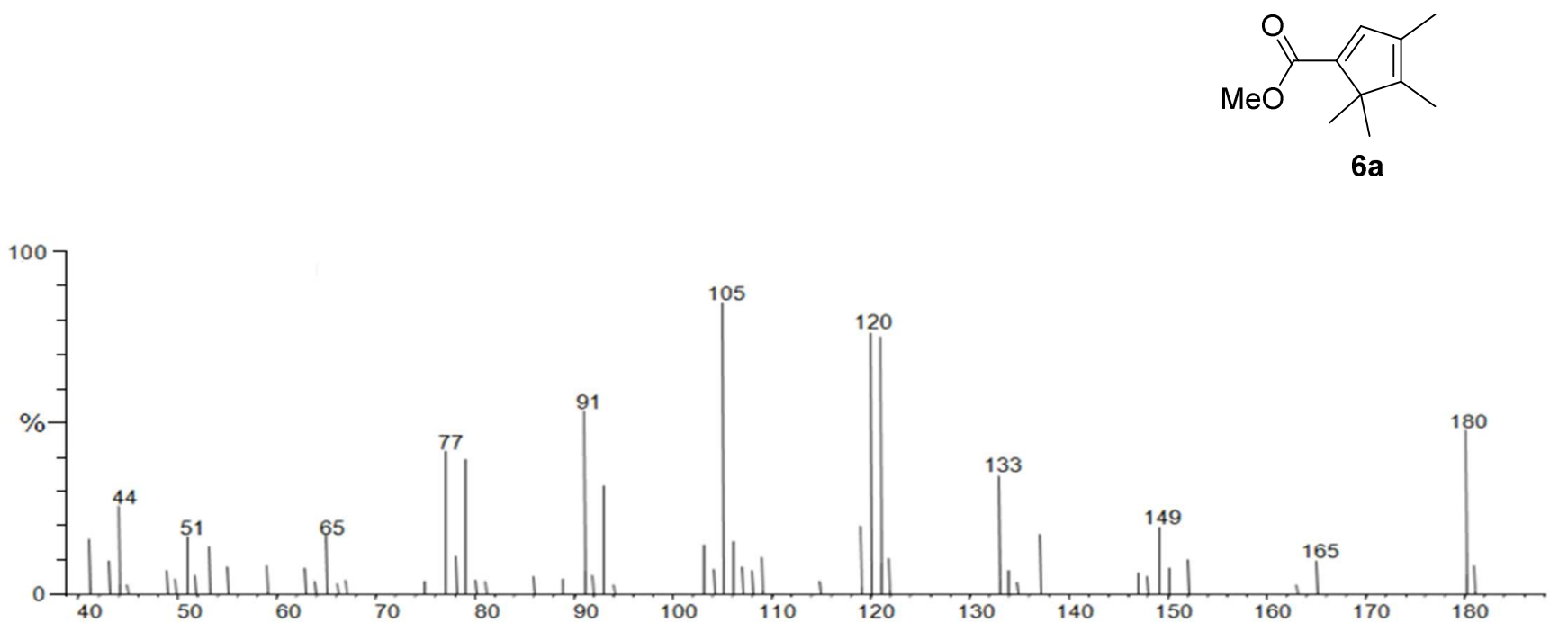

\begin{tabular}{|c|c|c|c|c|c|c|c|c|}
\hline Minimum: & 13.00 & & & & 0.0 & & & \\
\hline Maximum: & 100.00 & & 200.0 & 5.0 & 50.0 & & & \\
\hline Mass & $\mathrm{RA}$ & Calc. Mass & $\mathrm{mDa}$ & PPM & $\mathrm{DBE}$ & \multicolumn{3}{|c|}{ Formula } \\
\hline 165.0919 & 40.42 & 165.0916 & 0.3 & 2.1 & 4.5 & $\mathrm{C} 10$ & $\mathrm{H} 13$ & $\mathrm{O}$ \\
\hline 166.1001 & 16.59 & 166.0994 & 0.7 & $4 \cdot 3$ & 4.0 & $\mathrm{C} 10$ & $\mathrm{H} 14$ & $\mathrm{c}$ \\
\hline 167.1069 & 25.52 & 167.1072 & -0.3 & -1.8 & 3.5 & $\mathrm{C} 10$ & $\mathrm{H} 15$ & c \\
\hline 180.1144 & 13.04 & 180.1150 & -0.6 & $-3 \cdot 5$ & 4.0 & $\mathrm{C} 11$ & $\mathrm{H} 16$ & \\
\hline 181.1234 & 34.22 & 181.1229 & 0.5 & 3.0 & 3.5 & $\mathrm{C} 11$ & $\mathrm{H} 17$ & 0 \\
\hline
\end{tabular}

Figure S15. HREIMS of compound $\mathbf{6 a}$. 


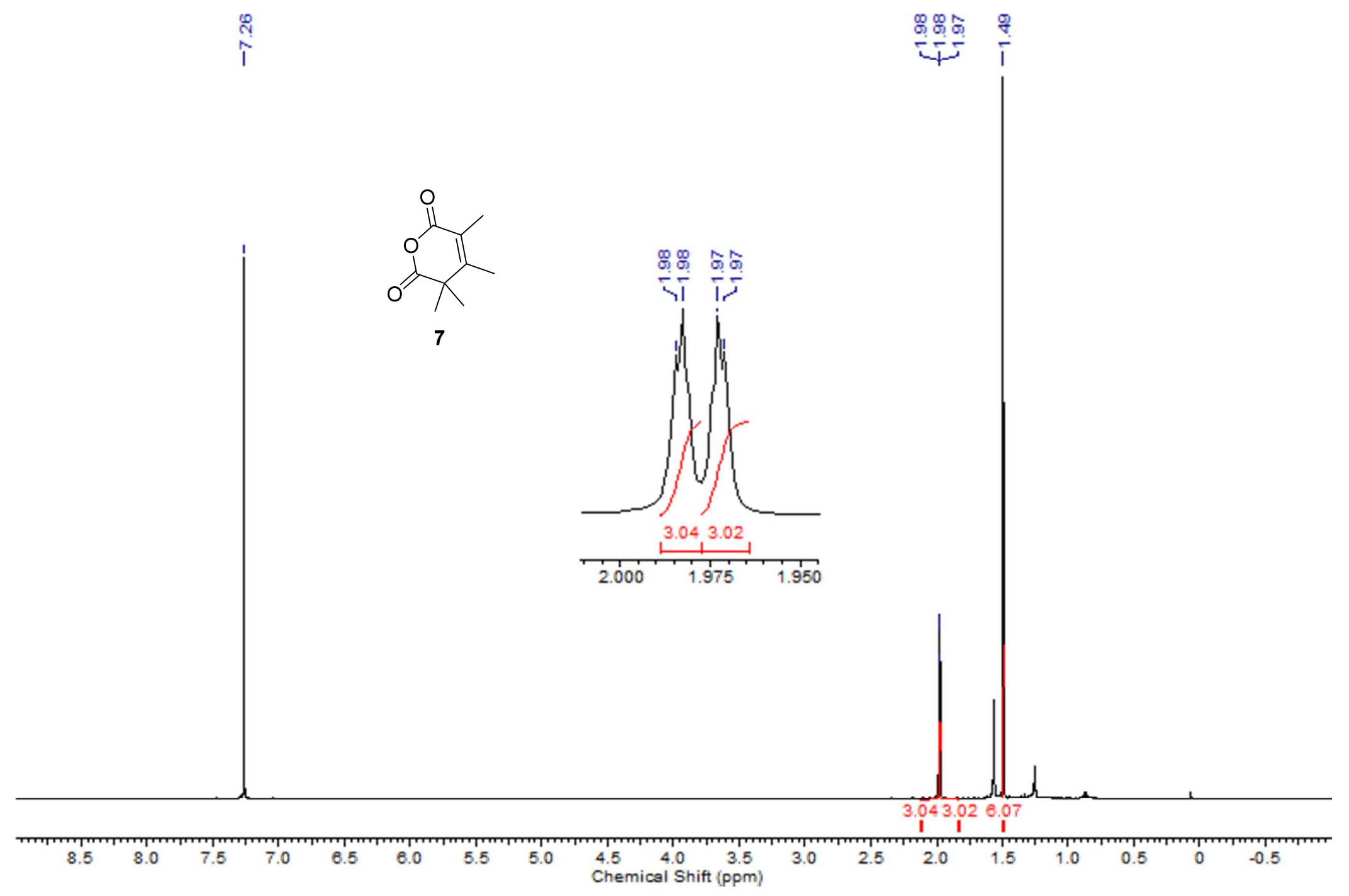

Figure S16. ${ }^{1} \mathrm{H}-\mathrm{NMR}$ spectrum of compound $7\left(\mathrm{CDCl}_{3}, 500 \mathrm{MHz}\right)$. 


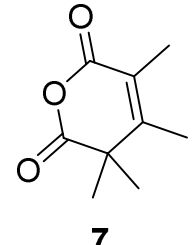

7

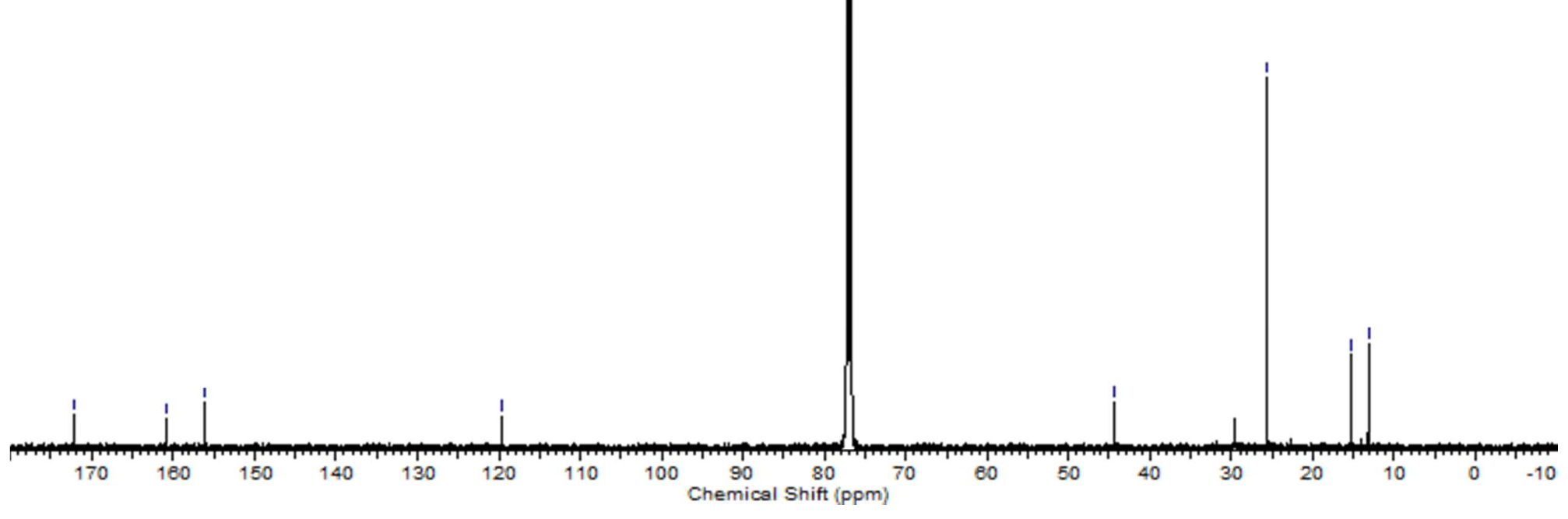

Figure S17. ${ }^{13} \mathrm{C}-\mathrm{NMR}$ spectrum of compound $7\left(\mathrm{CDCl}_{3}, 125 \mathrm{MHz}\right)$. 


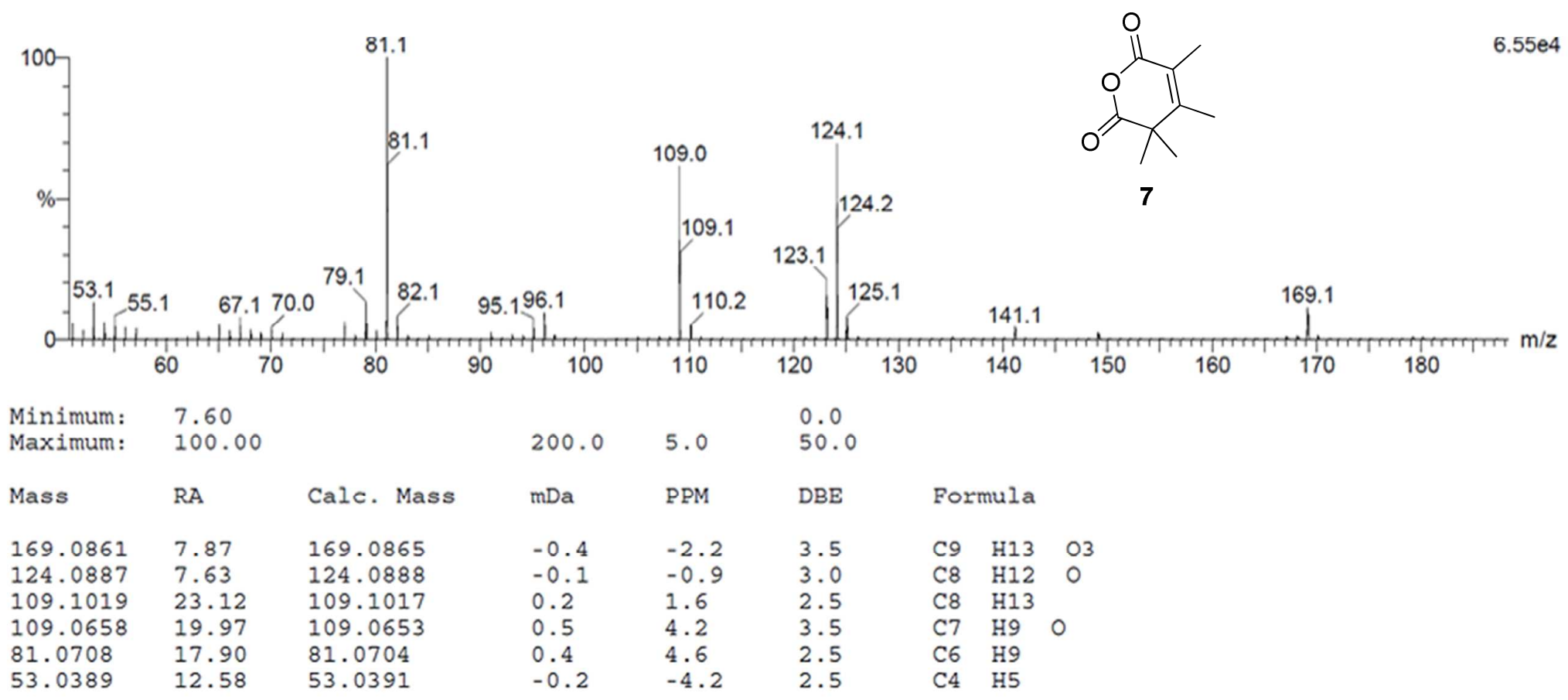

Figure S18. HREIMS of compound 7. 


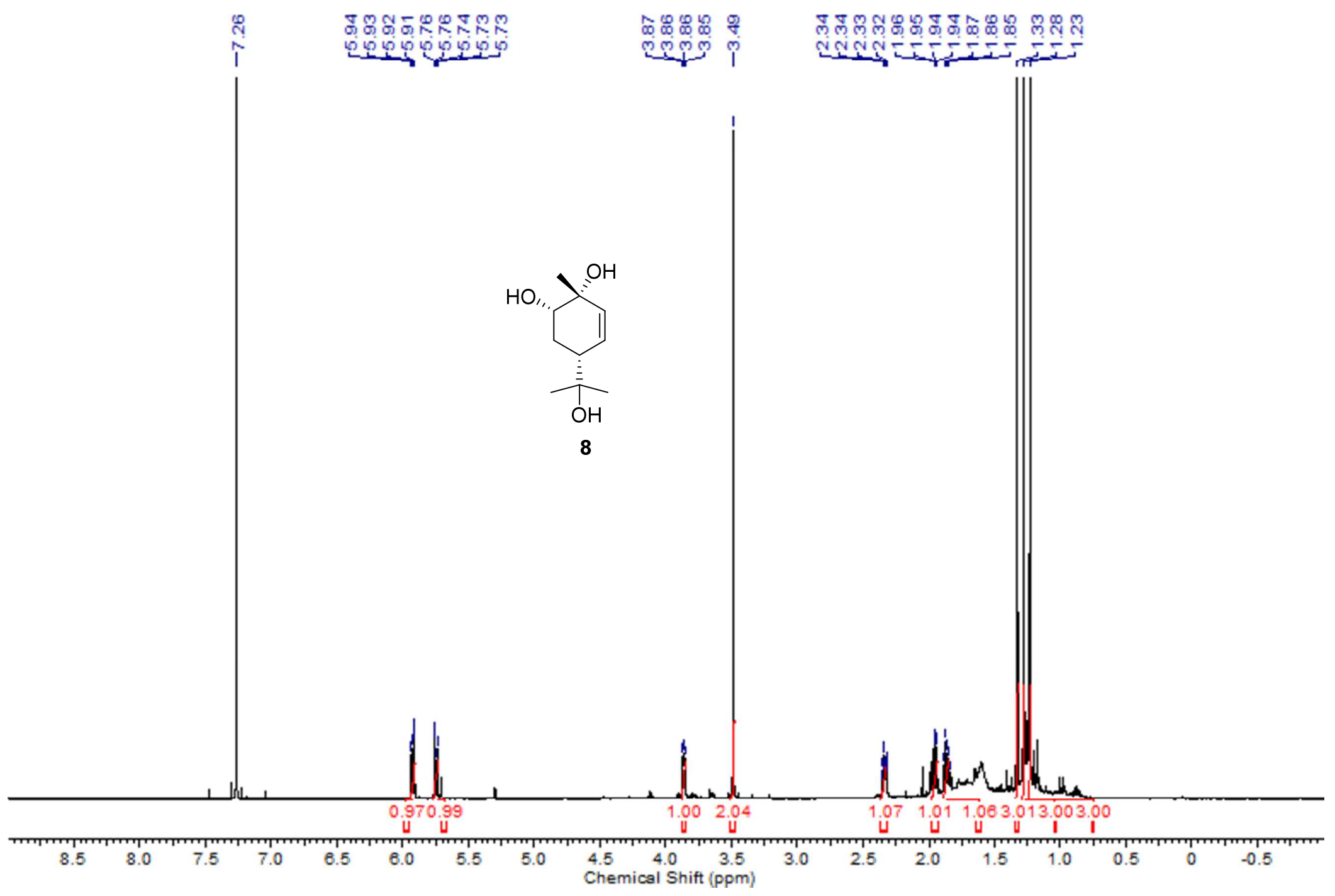

Figure S19. ${ }^{1} \mathrm{H}-\mathrm{NMR}$ spectrum of compound $\mathbf{8}\left(\mathrm{CDCl}_{3}, 500 \mathrm{MHz}\right)$. 


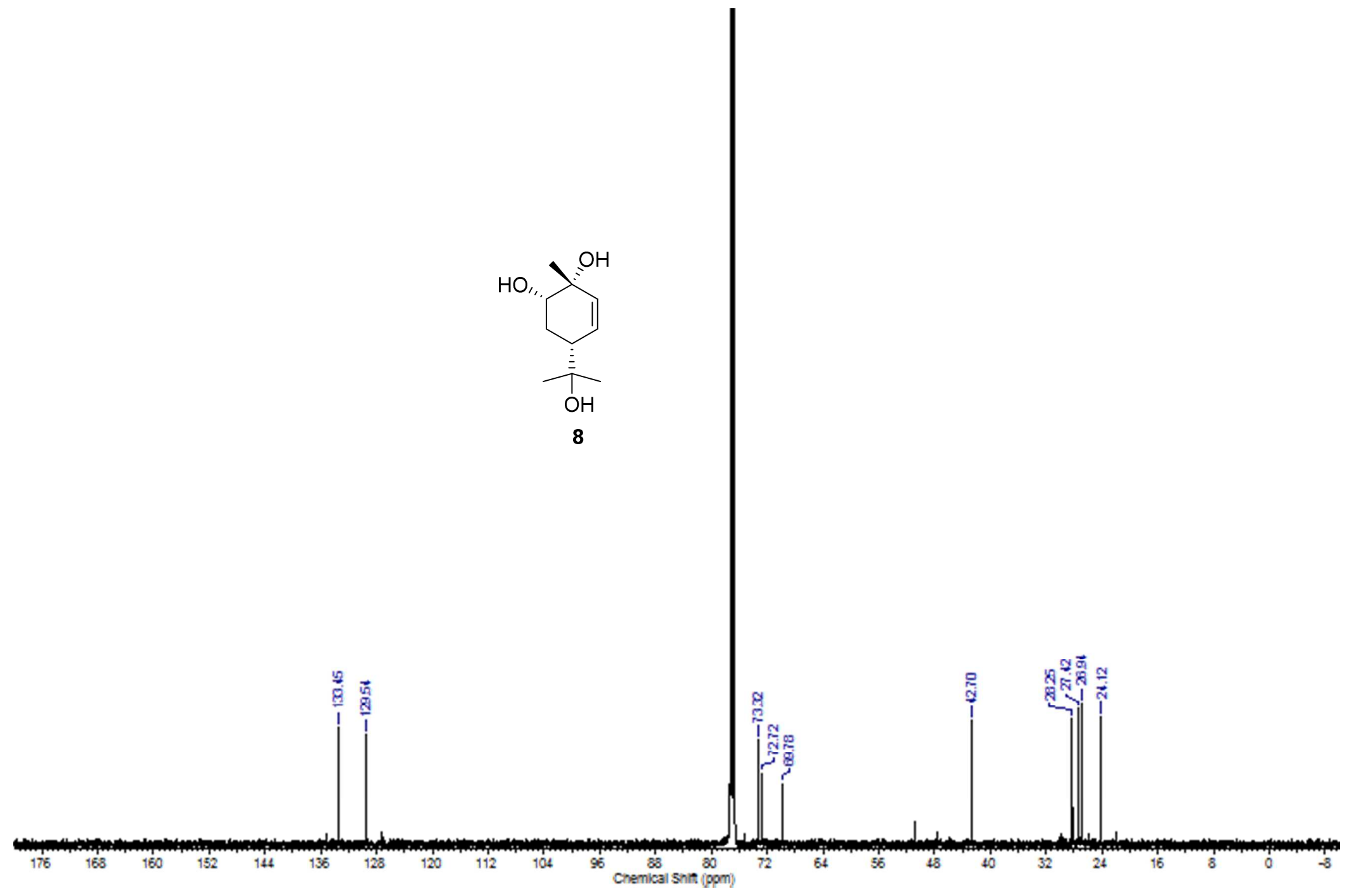

Figure S20. ${ }^{13} \mathrm{C}-\mathrm{NMR}$ spectrum of compound $\mathbf{8}\left(\mathrm{CDCl}_{3}, 125 \mathrm{MHz}\right)$. 


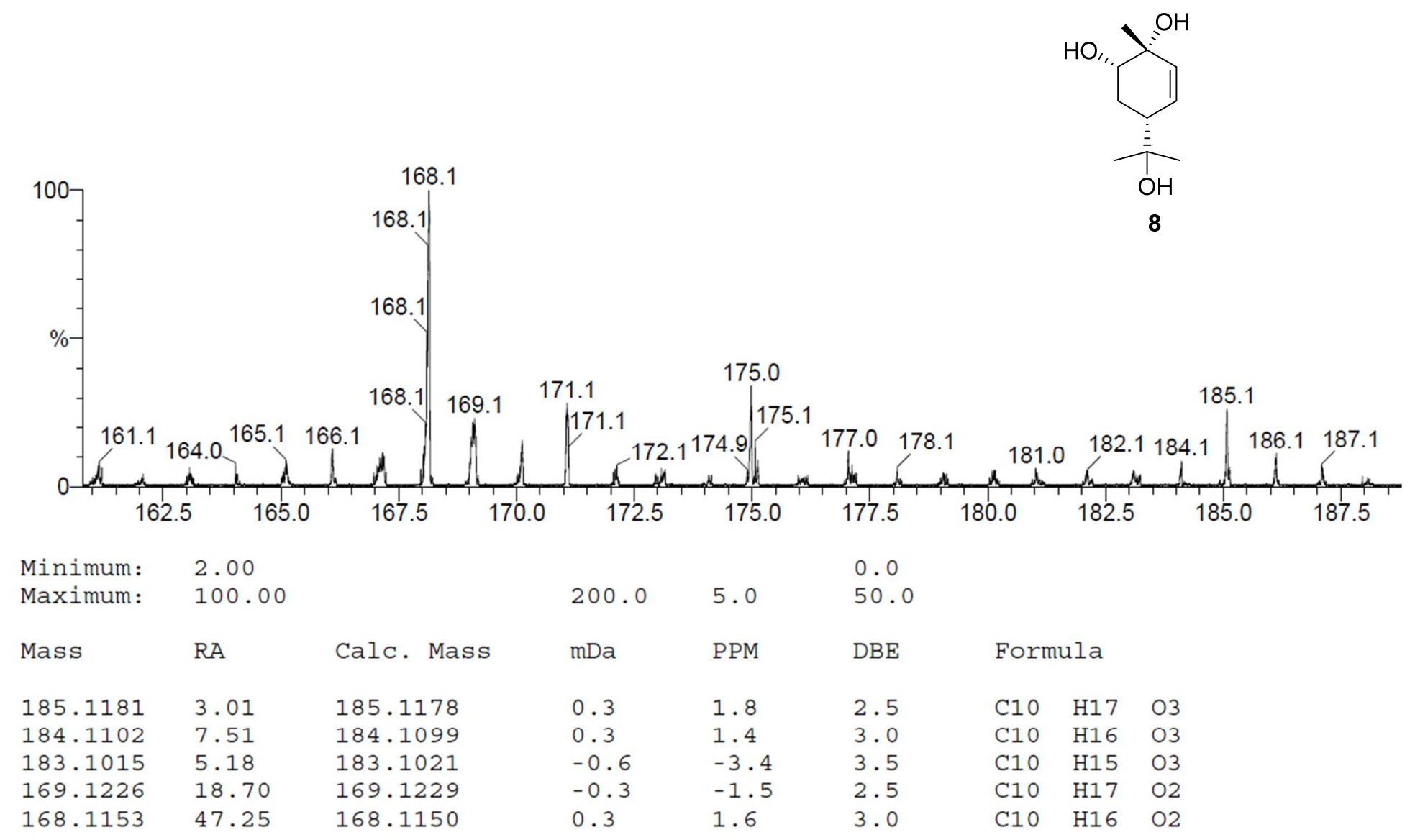

Figure S21. HREIMS of compound 8. 
Table S1. Phytotoxic Effects of Lavandula. luisieri extracts

\begin{tabular}{|c|c|c|c|c|c|c|c|c|}
\hline \multirow{3}{*}{ extract } & \multirow{3}{*}{$\mathrm{mg} / \mathrm{mL}$} & \multicolumn{3}{|c|}{ Lactuca sativa } & \multicolumn{4}{|c|}{ Lolium perenne } \\
\hline & & \multicolumn{2}{|c|}{ germination } & \multirow{2}{*}{$\begin{array}{c}\text { growth } \\
\text { root }\end{array}$} & \multicolumn{2}{|c|}{ germination } & \multicolumn{2}{|c|}{ growth } \\
\hline & & $24 \mathrm{~h}$ & $144 \mathrm{~h}$ & & $72 \mathrm{~h}$ & $168 \mathrm{~h}$ & leaf & root \\
\hline essential oil & 0.40 & $46.4 \pm 13.1^{b}$ & $100 \pm 0.0$ & $29.4 \pm 2.3^{b}$ & $50.0 \pm 18.1^{b}$ & $100 \pm 0$ & $68.7 \pm 7.0^{b}$ & $59.6 \pm 5.0^{b}$ \\
\hline hexane & 0.40 & $0^{b}$ & $20.0 \pm 4.1^{b}$ & $n a$ & $64.0 \pm 20.6^{b}$ & $84.6 \pm 5.4$ & $82.0 \pm 11.3$ & $67.2 \pm 8.2^{b}$ \\
\hline EtOAc & 0.40 & $7.5 \pm 2.5^{b}$ & $85.0 \pm 2.9$ & $50.8 \pm 9.5^{b}$ & $100.0 \pm 12.6$ & $97.4 \pm 5.7$ & $71.0 \pm 6.5^{b}$ & $79.4 \pm 5.9^{b}$ \\
\hline $\mathrm{EtOH}$ & 0.40 & $3.4 \pm 3.5^{b}$ & $22.5 \pm 7.5^{b}$ & $n a$ & $50.0 \pm 11.7^{b}$ & $100 \pm 0.0$ & $68.6 \pm 10.1^{b}$ & $80.7 \pm 9.5$ \\
\hline \multirow[t]{2}{*}{ aqueous } & $100 \%^{a}$ & $0.0 \pm 0.0^{b}$ & $0.0 \pm 0.0^{b}$ & $n a$ & $0.0 \pm 0.0^{b}$ & $0.0 \pm 0.0^{b}$ & na & na \\
\hline & $50 \%{ }^{a}$ & $0.0 \pm 0.0^{b}$ & $12.5 \pm 4.8^{b}$ & $n a$ & $0.0 \pm 0.0^{b}$ & $0.0 \pm 0.0^{b}$ & na & $n a$ \\
\hline anic fraction & 0.40 & $22.2 \pm 14.3^{b}$ & $87.5 \pm 4.8$ & $122.4 \pm 15.0$ & $22.2 \pm 19.1^{b}$ & $93.8 \pm 10.4$ & $84.9 \pm 10.3$ & $90.9 \pm 10.2$ \\
\hline
\end{tabular}

${ }^{a}$ Dilutions. ${ }^{b} \mathrm{p}<0.05$ Mann Whitney U-test (germination and growth). $n a$, insufficient number of roots available. 
Table S2. Antifeedant and Nematicidal Effects of Lavandula. luisieri extracts

\begin{tabular}{|c|c|c|c|c|c|}
\hline \multirow[b]{2}{*}{ extract } & concentration & Meloidogyne javanica & concentration & $\underline{\text { Spodoptera littoralis }}$ & Myzus persicae \\
\hline & $(\mathrm{mg} / \mathrm{mL})$ & $(\mathrm{J} 2 \text { mortality } \%)^{a}$ & $(\mu \mathrm{g} /$ disk $)$ & $\mathrm{FI}^{b}$ & $\mathrm{SI}^{b}$ \\
\hline essential oil & 1.0 & $9.0 \pm 1.0$ & 100 & $47.0 \pm 9.0$ & $58.0 \pm 8.0$ \\
\hline hexane & 1.0 & $14.2 \pm 3.1$ & 100 & $38.9 \pm 15.1$ & $61.8 \pm 6.9^{d}$ \\
\hline EtOAc & 1.0 & $10.0 \pm 6.7$ & 100 & $25.9 \pm 14.4$ & $71.2 \pm 6.8^{d}$ \\
\hline $\mathrm{EtOH}$ & 1.0 & $17.3 \pm 2.2$ & 100 & $64.0 \pm 11.0$ & $42.0 \pm 10.1$ \\
\hline aqueous & $100 \%^{c}$ & $100 \pm 0.0$ & - & - & - \\
\hline & $25 \%$ & $48.9 \pm 3.9$ & - & - & - \\
\hline & $12.5 \%$ & $2.2 \pm 0.6$ & - & - & - \\
\hline rganic fraction & 1.0 & $7.4 \pm 1.4$ & 100 & $37.8 \pm 16.0$ & $n t$ \\
\hline
\end{tabular}

${ }^{a}$ Corrected according to Scheider-Orelli's formula. ${ }^{b}$ Percent feeding or settling inhibition. $\left(\% \mathrm{FI} / \% \mathrm{SI}=\left[(1-(\mathrm{T} / \mathrm{C}))^{*} 100\right]\right.$ where $\mathrm{T}=$ consumption $/$ settling on treated disk; and $\mathrm{C}=$ consumption / settling on control disk. ${ }^{c}$ Dilutions. ${ }^{d} \mathrm{p}<0.05$, Wilcoxon signed-rank test. $n t$, not tested. 